\title{
The Impact of Long-term Care Needs on the Socio- economic Deprivation of Older People and Their Families: a Scoping Review Protocol
}

\section{Rossella Martarelli}

Istituto Nazionale di Riposo e Cura Anziani V E II Istituto di Ricovero e Cura a Carattere Scientifico:

INRCA-IRCCS https://orcid.org/0000-0003-4838-6494

\section{Georgia Casanova ( $D$ G.Casanova@inrca.it)}

IRCCS-INRCA National Institute of Health \& Science on Ageing, Centre for Socio-Economic Research on Ageing and Instituto de Investigación en Políticas de Bienestar Social (POLIBIENESTAR) - Research Institute on Social Welfare Policy, Universidat de València https://orcid.org/0000-0002-3944-873X

\section{Giovanni Lamura}

Istituto Nazionale di Riposo e Cura Anziani V E II Istituto di Ricovero e Cura a Carattere Scientifico: INRCA-IRCCS

\section{Protocol}

Keywords: older people, family caregivers, long-term care, informal care, socio-economic deprivation, catastrophic health expenditure, out-of-pocket spending

Posted Date: September 21st, 2021

DOI: https://doi.org/10.21203/rs.3.rs-816117/v1

License: (c) (1) This work is licensed under a Creative Commons Attribution 4.0 International License. Read Full License 


\section{Abstract}

\section{Background}

Population ageing, constantly on the increase in all countries worldwide, has long been the object of scientific research from several perspectives, including multi and interdisciplinary approaches. This scoping review aims to investigate the socio-economic consequences of older people's poor health on their own economic conditions and those of their families. This study aims to: a) map the main concepts that characterise the body of literature pertaining to this issue; b) identify conceptual gaps or unexplored research areas to be addressed; $c$ ) delve into the ways of arguing about the difficulties that affect a large number of families with older members to care for, especially with regard to the concept of socioeconomic deprivation, which in our perspective includes both material and social deprivation (e.g. in the form of loneliness experienced as a consequence of health disorders). This protocol fulfils the purpose of clarifying the stages and methods of the study and listing the techniques used.

\section{Methods}

This article is being drafted according to the Preferred Reporting Items for Systematic reviews and MetaAnalyses for Protocols 2015 (PRISMA-P 2015). The rationale behind the study and its stages are aligned with the guidelines of Lockwood et al. (2019) and the recommendations of Munn et al. (2018):

Each stage links up with the next, according to the Preferred Reporting Items for Systematic reviews and Meta-Analyses extension for Scoping Reviews (the 2020 PRISMA Statement), while the reporting phase refers to the Joanna Briggs Institute (JBI) checklist. The search process is being performed by means of databases such as PubMed, Scopus and Web of Science. The latest version of MAXQDA will be used for analyzing all data.

\section{Discussion}

We aim to highlight and connect the most useful insights addressed to stakeholders and policymakers and, most of all, the ones valuable to social innovation. Nevertheless, it is necessary for us to remark that, despite the prevalence of the English language, most research articles are written and published in other languages. Therefore, they are excluded from the search process.

Systematic review registration

Open Science Framework (OSF), https://osf.io/xq58z

Registration DOI: 10.17605/OSF.IO/XQ58Z

\section{Background}


The global population is getting older and older. In 2020, people aged at least 65 represented $9.3 \%$ of the global population, and they are expected to increase to $16.0 \%$ by 2050 (1). The oldest segment of the population, in particular, is strongly on the rise: the so-called oldest-old (those over 80 years old) account for $6.3 \%$ of the total population in Europe, and their incidence is expected to double by 2050 (2) (3). The number of older people in need of long-term care (LTC) is set to grow radically worldwide, together with the request for formal and informal care (4). Caring for the state of health of older people involves interventions at the biomedical and psychosocial level and measures of economic interest (5), and all of them weigh on the balance of families (see Graph 1, titled "Research concepts and goals"). In particular, long-term care has been included among the highest priorities of national and international policies (6).

Many international research projects (e.g. EUROFAMCARE, ANCIEN, INTERLINKS, MOPACT, and Cequa) focussed their attention on LTC and its different aspects and actors. Since they are the main caregivers (see the meaning of the term "caregivers" in Graph 1), families represent one of the most important stakeholders in the LTC context (7) (8) (9). In 2017, after analyzing the situation of many countries, Muir et al. (10) (11) pointed out that the cost of LTC was actually high compared with typical incomes. Even in countries with advanced social protection systems, family-givers'care-related out-of-pocket expenditure is not negligible at all; more and more families, in fact, buy private care services. In addition to direct costs, they are involved in difficulties derived from a variety of indirect costs: the more time they spend caring for older people, the less they work, and this easily results in a reduction in earning capacity (12) (13) (14).

There is no shortage of studies on the interplay between the socioeconomic deprivation of older people and their health conditions. Material deprivation reduces the possibility of coping with health needs and problems (15) (16) and influences both the psychosocial well-being (17) and the cognitive conditions of these people (18). Moreover, as indicated in many studies, significant economic impoverishment, together with socio-relational deprivation, creates remarkable differences in life expectancy, since wealthy seniors generally live longer (19) (20).

Nevertheless, it should be stressed that studies carried out in this field, while remarkable for the great attention paid to the effects of economic hardship on health conditions, often overlook the effects of health conditions on the socio-economic status (SES) of impaired or chronically ill people. Therefore, it seems to us that further studies on how health problems and related expenses affect the economic situation of families are necessary, especially to investigate the relationship between the type of approach to care provision for older adults with ADL limitations - both formal and informal care - and the socioeconomic deprivation of the older adults themselves and that of their families.

Guided by our intention to support research in this field and contribute to its dissemination, we decided to conduct a scoping review ( $\mathrm{ScR}$ ) that mainly aims to: 1) systematically scan and evaluate the literature developed on the issue of older adults who need to be provided with LTC and their SES; 2) search out any possible conceptual gap in the reference literature; 3 ) map the main existing concepts and open 
questions, especially in terms of socioeconomic deprivation due to the direct and indirect costs of LTC; 4) identify the types of outcomes; 5) synthesise the main accessible results.

This type of review and the method of application allow us to meet these objectives and to provide an upto-date summary on the state of the art of scientific research on this issue. Moreover, this study allows us to capture the meaning of the key concepts and definitions used in this field by researchers around the world; therefore, it enables us to test their level of use in following a multidimensional perspective which is, in our opinion, indispensable.

This scoping review is part of a cross-national research effort promoted by the Family International Monitor (FIM). The current research plan of FIM (21) aims to delve into the many ways in which a condition of deprivation - understood as material and social deprivation — can affect families worldwide. The study is also part of the EU-funded SEreDIPE project (Horizon 2020 MSCA-IF-2019 Grant Agreement No. 888102), focused on the economic impoverishment of families with impaired older people and managed by the University of Valencia. Both projects deal with the concepts of "family" and "deprivation" from a multidimensional perspective (22).

\section{Methods/design}

This study refers to a composite but coherent set of methodological indications that describe how to conduct a scoping review and when it is appropriate to do so. We refer to: a) the Lockwood et al. guidelines (23) on how to set up a scoping review; b) the Munn et al. recommendations (24) for structuring this type of study (recommendations highlighted in bold in Graph 1); c) the Preferred Reporting Items for Systematic reviews and Meta-Analyses extension for Scoping Reviews (25); d) the Joanna Briggs Institute (JBI) checklist (26).

These guidelines enable us to realize: a) all of the steps to be taken, i.e. 1) pre-planning; 2) protocol; 3) conducting and reporting; $b$ ) how to distinguish a scoping review from a traditional or systematic review, i.e. how to achieve all of the objectives and present the results; $c$ ) how to sequence all of the study's stages and sub-stages; $d$ ) the type of information to be provided, such as the types of sources or "effect measures" extracted from the selected studies (e.g. prevalence ratios for functional limitations; income inequality ratios; at-risk-of-poverty rate etc.).

The reporting phase described in the PRISMA Statement is congruent with the corresponding phase described in the JBI Manual. Finally, this protocol aligns with the Preferred Reporting Items for Systematic Reviews and Meta-Analyses for Protocols 2015 (the PRISMA-P Statement; see "Additional file 1").

Graph 2, titled "The study's methodological framework", consists of a detailed representation of the steps and sub-steps of this scoping review.

\subsection{Pre-planning}


This stage is based on the formulation of a series of broad questions:

1) What are the socio-economic conditions of families with impaired older people? 2) Is it true that most of these older people and their families are about to fall into ruin? 3) How can family caregivers cope with the economic burden of care for the old? In response to these initial questions, we agreed to: 1) identify and draw up the boundaries of the main concepts used, as well as any gaps traceable in the reference literature, in order to understand how the set of changing relationships between economic and health conditions is being explored and put into perspective; 2 ) investigate whether these families are so socioeconomically deprived that they really experience both a concrete economic impoverishment and social marginalization, or whether they have some social support to mitigate such pressing difficulties; 3 ) identify the types of evidence and tools for measuring the health care-seeking behaviour of older people in need and their health requirements.

\subsubsection{Brainstorming}

The brainstorming phase allowed us to: a) identify the most suitable type of study; b) adjust and improve all the points enumerated above.

To begin with, each author assessed the structuring level of our initial questions and goals independently of the others, based on the several available scoping reviews and his/her own previous knowledge; this was followed by a brainstorming session which helped us to discuss our opinions, especially in relation to the possibility of addressing systematic reviews. We were in agreement on the main lines, i.e. that such a set of non-stringent questions and goals could be appropriate for an extensive investigation of the reference literature. Moreover, when compared to the seven goals listed by Lockwood et al. (see Graph 1), our initial purposes turned out to be very appropriate for a scoping review. We were able to confirm that most of those seven goals could be pursued and that this type of study could be undertaken. The brainstorming phase, i.e. an in-depth debate about the extent to which our questions should be defined, also allowed us to refine these and generate a final list of questions, which are: 1) To what extent are such families hit by socioeconomic deprivation? 2) How much does the cost of care for the old contribute to their economic hardship? 3) How thoroughly does the reference literature deal with these issues? 4) What are the most commonly found definitions of the "socioeconomic deprivation" concept in the reference literature? Finally, our initial purposes were slightly revised and definitively fixed on the basis of these 'new' questions.

\subsubsection{Definitive aims}

This study aims to: 1) identify the main key concepts used; 2) pinpoint any little explored conceptual areas or countries where research needs to be strengthened; 3 ) examine how socioeconomic deprivation and related phenomena are conceptualized and debate whether the main definitions are consistent with our concept of "multidimensional deprivation"; 4) identify and report on the main existing results and insights provided, particularly those that stakeholders and policymakers can benefit from, e.g. in terms of 
social innovation. This broad scope of objectives allowed us to filter through as many studies and evidence as possible, regardless of the countries in which the studies were conducted.

\subsubsection{Initial keywords}

A list of keywords was also drawn up in the planning stage, which included:

Long-term care, older (elderly) people, caregiver, family caregiving, impoverishment, deprivation, socioeconomic deprivation, economic, economic impact, poverty, and multidimensional poverty, since many researchers frequently use the term "poverty" as a synonym for "socioeconomic deprivation" in their articles. As detailed in the chapter referring to the "Protocol" (which describes the entire search process as well as the first exploration), these keywords were used to begin investigating all the relevant studies.

\subsection{Protocol}

The protocol phase consists of: 1) a detailed explanation of the study phases, which is precisely the purpose of drafting this document (see the following paragraph "Writing the protocol"); 2) a detailed explanation of the selection process: which studies and related research articles are concretely sought? What criteria do we refer to during the selection process? Since this is a scoping review, choosing broad criteria is strictly necessary (as shown in Graph 2); therefore, we are only required to define three criteria:

a) participants; b) concepts; c) context (detailed in the paragraph "Selection process and eligibility criteria"); 3) a detailed list of any information related to the study's development (see the paragraph "Scoping review and evidence source details").

\subsubsection{Writing the protocol}

This protocol is being drafted in accordance with the aforementioned guidelines. It mainly aims to illustrate the study's methodological framework and the types of materials and data to be used or obtained, such as: variables to be analyzed, gaps to be identified, and summary charts to be created (see Table 2). To this end, every effort has been made to make the study's content clear, methodologically reliable and adequately planned. The protocol also clarifies the link between questions and objectives (see previous paragraphs). Finally, it aims to describe how the unfinished steps will be completed.

\subsubsection{Selection process and eligibility criteria}

Thus far, all articles were imported from the following databases (sorted by number of items found): PubMed, Scopus, and Web of Science. We also consulted the Wiley Online Library, which provided some useful references. All data is stored in cloud-based archives (Mendeley/EndNote X9). The participants, concepts, context (PCC), and eligibility criteria that underpin this study are described below.

Participants: older adults in need of LTC and their family caregivers (both the family members who live with the older people and the ones who provide indirect assistance are included). We refer, in particular, to the so-called oldest-old people who cannot perform routine activities of daily living or self-care, regardless of their type of health problems. Moreover, the number of participants is not limited by the type of family 
or the country in which they live; both single-parent families (e.g. older people living alone) and married couples, with or without adult children, are included.

Concepts: a) the socioeconomic deprivation of impaired older people provided with LTC; b) the socioeconomic deprivation of their families. In detail:

1) "Older adults in need of (or provided with) LTC" are the ones affected by a reduced ability to perform routine activities, such as washing or bathing, dressing, feeding, transferring, and mobility, owing to agerelated functional decline or chronic health problems. The fact that they depend on the treatment they need makes LTC indispensable; 2) LTC refers to services that help people in need of care, especially the old, with their physical and emotional needs over an extended period of time. LTC is by far the greatest health-care need of older adults and includes informal care (27); 3) "informal care" is generally referred to as "unpaid care", mainly provided by a person connected to the older adult being taken care of, e.g. a spouse, child, or other relative. Nevertheless, the growing need for care among home-dwelling older adults is leading to the involvement of a higher number of people to support the old; 4) the term "family caregiving" represents the situation in which older adults are cared for by one or more family members, defining families in the broad sense, i.e. as individuals with a specific personal (see Graph 1). Family caregivers, in fact, can manage and provide home-based LTC both directly and indirectly; 5) the term "socioeconomic conditions" synthesizes a host of detectable factors, i.e. family assets, income, in workbenefits (if any), and savings and social ties related to the socio-demographic characteristics of family members. All of these are elements to which we must attribute the worsening or containment of LTC's economic burden. In the case of illness, the combined effect of a shortage or absence of these factors may alternatively lead to: a) "catastrophic health expenditure", which occurs when out-of-pocket health expenditure exceeds a certain ratio of household income to health spending); b) "economic impoverishment", which occurs when average household consumption falls below the international or national poverty threshold as a result of care expenses.

Context: all types of long-term care services for older people, especially informal care, in all geographical areas (both western and non-western countries). Nevertheless, the search process is conditioned by the considerable number of relevant studies conducted in low- and middle-income countries (respectively: LICs and MICs).

Eligibility criteria: in addition to the basic criteria illustrated above (PCC), it must be specified that: a) all studies investigating factors and policies that link activities, situations, or conditions such as old age, poor health, long-term care, health-related behaviours, and socioeconomic deprivation of families with older members affected by ADL limitations are included; $b$ ) studies proposing solutions to the economic problems triggered by health needs are particularly taken into account; $\mathrm{c}$ ) special consideration is given to the ones outlining socially innovative solutions; d) all types of quantitative studies are included; e) qualitative studies and mixed methodologies, although scarce, are also included; f) all types of countries are considered in order to see whether there are any methodological differences that are somehow linked to the specific type of context; g) primary and secondary studies are considered; among the latter, 
systematic and scoping reviews are also taken into account; $h$ ) all selected relevant articles are no older than five years; exceptions to this rule are those selected due to the pertinence of the sources, of up to a maximum of ten years; i) all selected articles are to be written in English; j) empirical publications in peerreviewed journals are preferred.

\subsubsection{Scoping review and evidence source details}

The features characterising the scoping review and the sources referred to can be summarised as follows:

a) Scoping review title: Ageing, Long-Term Care, Poverty, and Socioeconomic Deprivation of Families: results from a scoping review;

b) Types of evidence sources: scientific journals; mainly research articles;

c) Details of evidence sources: we will list a number of items such as authors, publication dates, journal titles, volumes, and materials (if any) attached to the selected articles. All this information will be published at the end of the selection process;

d) The year of publication of the most recent sources among those selected: 2020;

e) Countries: we will also compile a list of countries where the selected studies were carried out. All this information will be published at the end of the selection process;

f) Types of evidence: most of the findings that we typically expect result from quantitative studies, often reinforced by the application of indicators (e.g. of disability or socioeconomic deprivation). For the most part, these studies turn out to be conducted on the basis of secondary data, resulting from both crosssectional and longitudinal studies. Qualitative studies are uncommon. Measurements of the probability of experiencing genuine economic impoverishment and social exclusion (after adjusting for age and income), as well as the degree of association between socio-relational deprivation and health problems, are generally the most common outputs;

g) Concepts and context of selected studies: the ways in which it is possible to care for older people in need of LTC in all countries, especially in middle- or low-income countries (MICs; LICs). The types of caregiver activities and the types of health problems of assisted persons are not indicated (bar a few exceptions). These studies mainly delve into: 1 ) the different shortcomings of national social protection systems; 2 ) the economic consequences that affect families; 3 ) the role played by social skills in order to avoid falling into material and social deprivation;

h) Participants in the selected studies: 1) caregivers: spouse or adult children (especially the ones cohabiting with the persons who need to be cared for, but this is not always specified); 2) care recipients: old, old-old and oldest-old impaired people; 3 ) families: all types of families (including those with adult children living elsewhere, although this is not clearly specified). 
i) Details and results to be extracted from evidence sources:

1) Measurement properties of Activities of Daily Living scales (ADLs) used by most national surveys (28) (29), e.g. need assistance, received assistance, duration, special equipment, and perceived level of difficulty;

2) Items and measurement properties of the Frailty Index used by some of the selected studies (56-items $\mathrm{FI})$, e.g. disabilities, self-reported health conditions, hearing, eyesight, cognitive function, and depressive symptoms;

3) Measurement properties of the Index of Multiple Deprivation 2004, used to identify the most and the least deprived areas in the UK.

\subsection{Conducting and reporting}

This phase includes all the actions carried out or to be carried out at the operational level, from the beginning to the end of the study. Therefore, it also refers to the outputs of our first exploratory investigation.

This initial step allowed us to identify the keywords we needed to use to perform a second selection, both to make a selection of chosen articles and to select new ones. A total of over ten new keywords were identified, as illustrated in Table 2. We identified four main thematic areas to refer to in order to search for the most useful keywords: 1) family; 2) older people to be cared for; 3) assistance; 4) socioeconomic deprivation. Most of these (new) keywords pertain to the fourth area, e.g. healthcare expenditure, spending, payments, economic impoverishment/costs, burden, socioeconomic status, and social differences. With regard to the first and the third, we added household and informal (home) care, respectively (see Table 1 below which represents all the combinations of keywords we are using). 


\section{PubMed ((caregiver[Title]) AND (poverty[Abstract])) OR (socioeconomic deprivation[Abstract])) AND (older people[Abstract])}

\section{Filters (2): Abstract; Journal}

2. Wiley O.L "intergenerational" anywhere and "ageing" anywhere and "expenditure" anywhere published in the "Australasian

Journal on Ageing" — Filters: not applied.

3. PubMed ((("2019/01/01"[Date - Publication] : "2019/12/31"[Date - Publication])) AND (long term care[Title])) AND

(socioeconomic deprivation[Abstract])) OR (poverty[Abstract]) - Filters (5): Abstract; Journal Article; English;

MEDLINE; Aged: 65 + years.

4. Cambridge keywords to enter: ageing - generational - spending - family

Univ. Press Filters (3): Journal "Ageing \& Society"; 2016-2021; "only show content I have access to".

5. Scopus (ABS(expenditure) AND KEY (older AND people) AND ABS (family)) - Filters: not applied.

6. PubMed ((poverty) AND (older people)) AND (informal care[Title/Abstract])) OR (home care[Title/Abstract])

Filters (6): Article; Last 5 years; English; MEDLINE; Aged 65+; 80 and over.

7. Web TOPIC: (impoverishment) ANDTOPIC: (household) ANDTOPIC: (caregiver) OR TOPIC: (deprivation) OR TOPIC:

of Science

(poverty) AND TOPIC: (elderly) AND TOPIC: (aged) AND DOCUMENT

TYPES: (Article) AND LANGUAGE (English)

Categories: (health care sciences services OR sociology OR health policy services OR social issues).

8. PubMed ((family caregiver[Title/Abstract]) AND (socioeconomic deprivation[Title/Abstract])) OR (poverty[Title/Abstract])

Filters (5): Article; Last 5 years; English; Aged: 65+; 80 and over.

9. Scopus (TITLE-ABS-KEY ("older AND people" OR elderly) AND TITLE-ABSKEY ("household AND impoverishment") OR ABS

(deprivation) AND KEY (economic)) AND (LIMIT-TO (SUBJAREA,"MEDI") OR LIMIT TO (SUBJAREA,"SOCI")).

10. PubMed (((home care[Title/Abstract]) OR (informal care[Title/Abstract])) AND (older people[Title/Abstract])) OR 


\section{PubMed (((caregiver[Title]) AND (poverty[Abstract])) OR (socioeconomic deprivation[Abstract])) AND (older people[Abstract])}

\section{Filters (2): Abstract; Journal}

(elderly[Title/Abstract])) AND disability[Title/Abstract]) - Filters (7): Article; last 5 years; English; 80+; 45+; 45-64; 65+.

11. PubMed (("Age and ageing"[Journal]) AND (long term care[Title])) AND (socioeconomic deprivation[Title/Abstract])) OR

(poverty[Title/Abstract]) — Filters (4): Journal Article; from 2019/1/1 to 2019/12/31; English; MEDLINE.

12. Wiley O.L "'"informal care" OR "home care"'" anywhere and "'"older people" OR

"elderly"'! anywhere and "carers" in Abstract

Filters (3): 2012-2021; Health\&Health care; Journals.

13. Wiley O.L "'"informal + care"+OR+"home + care"'" anywhere and "'"older people" OR "elderly"'! anywhere and "carers" in Abstract

published in "Health Economics" published in "Australasian Journal on Ageing" published in the "Scandinavian Journal of Caring Sciences" — Filters: not applied.

14. Wiley O.L "economic+" anywhere and "'"older people" OR "aged"' anywhere and "family" anywhere and

"caregivers" in Abstract and "intergenerational" anywhere — Filters: not applied.

15. Wiley O.L "'"poverty"+OR+"multidimensional poverty"' in Abstract and "health" OR "informal care" OR "long term care"

in Abstract — Filters: 2015 - 2010.

16. PubMed (("Australasian journal on ageing"[Journal]) AND (intergenerational)) OR (ageing)) AND (costs)

Filters(4): Journal Article; time spam: from 2016/1/1 to 2019/12/31; English; MEDLINE.

17. PubMed (((poverty[Title/Abstract]) OR (multidimensional poverty[Title/Abstract])) AND (disability[Title/Abstract])) OR

(functional limitations[Title/Abstract])) AND (low-income countries) - Filters (3): article; 1/1/2017-31/12/19; English.

18. Wiley O.L "'informal care" OR "long term care"'" in Abstract and "family" anywhere and "''aged" OR "elderly"'I in Abstract

and "carers" anywhere - Filter (1): 2015/2019.

19. PubMed $\quad((((($ disability[Title]) AND (poverty[Title/Abstract] $))$ OR (deprivation[Title/Abstract])) OR

(economic costs[Title/Abstract])) AND (older people[Title/Abstract])) OR (elderly[Title/Abstract])) AND (low income) 


\section{PubMed (((caregiver[Title]) AND (poverty[Abstract])) OR (socioeconomic deprivation[Abstract])) AND (older people[Abstract])}

Filters (2): Abstract; Journal

Filters (3): Articles; last 10 years; English.

20. PubMed (((poverty[Title]) OR (multidimensional poverty[Title])) OR (deprivation[Title])) AND (age[Title/Abstract])) OR

(ageing[Title/Abstract]) — Filters (5): Article; English; 01/01/2019-present; 65 + and $80+$ years.

21. PubMed $\quad(((($ socioeconomic[Title]) OR (socio-economic[Title])) AND (health[Title/Abstract])) OR (health problems[Title/

Abstract])) AND (care[Title/Abstract])) OR (ADL limitations[Title/Abstract]) — Filters (3): Article; English; last 10 years.

22. PubMed (((life expectancy[Title/Abstract]) AND (social differences[Title])) OR (elderly[Title])) AND (socioeconomic

status[Title/Abstract]) - Filters: 45-64 years; aged: 65 + years; time span: from $01 / 01 / 19$ to $31 / 12 / 2019$.

23. PubMed

$(((($ poverty[Title]) OR (healthcare expenditure[Title] $))$ AND (income[Title/Abstract])) OR (low-income

countries[Title/Abstract])) AND (deprivation[Title/Abstract])) OR (payments[Title/Abstract])

Filters (3): from 01/01/2019 to 31/12/20; article; English.

24. PubMed ((((family caregiver[Title]) OR (older[Title])) AND (burden[Title/Abstract])) OR (socioeconomic

status[Title/Abstract])) AND (activities of daily living[Title/Abstract])) OR (functional limitations[Title/Abstract])

Filters: from 01/01/2019 to 31/12/20; article; English.

A) articles or books from non-digital archives: 1 article from the journal "On Intimacies: cultures and practices in current societies" - Issue 3/2017: working carers + 1 book); B) [Internet]: keywords: "abstract"; "family caregiving"; "economic impact". [Book: 1]; C) JSTOR: "Proceedings. Annual Conference on Taxation and Minutes of the Annual Meeting of the National Tax Association" Filters (3): 2018-2021 + "only Journals" + refined by: "Family caregiving".

\subsubsection{Initial selection outputs}

Thirty-five research articles were initially selected using PubMed and Wiley Online Library as a starting point, followed by Scopus and Web of Science. This first selection also includes studies concerning the relationship between material and social deprivation and self-perceived well-being, or the connection between an early withdrawal from working life and family caregiving. The last articles offer us many 
insights into the economic situation of women in particular and the level of decrease in earning capacity of family caregivers in general. In any case, most of the selected articles deal with the issue of health inequalities among older people; others emphasize that it is easily possible to fall into material deprivation even in high-income countries (HICs) when faced with serious health problems. It also does not seem to us to be insignificant that some of the first articles we found indicated that there is urgent need to thoroughly revise the methods and techniques for measuring the impact of ageing on the economic situation of countries.

\subsubsection{Second selection}

The additional keywords mentioned above turned out to be highly suitable as selection tools, since this second step allowed us to find many other relevant studies.

Together with those selected in the previous step, 63 research articles could be counted so far. The articles we are now finding are more focused on family economic difficulties and on how families act to ward off any type of economic impoverishment. What is striking is the fact that many older people around the world give up care or reduce it significantly.

The drawing of a flow chart is planned in order to represent the entire search process in our final article. This graph will show how many articles were definitively selected, as well as how many were rejected and why.

\subsubsection{Quality assessment: data extraction}

Once the search process is finalised, we will begin to take note of the studies' main features with the intention of identifying the extent to which the studies explain their aims, methods, and results. This process, which is referred to as "quality assessment", consists of two sub-stages: 1) data extraction: data will initially be extracted as text variables using a modified JBI data extraction instrument (see additional file 2). All the main features of each article will be summarized on the basis of a classification grid made up of the following items: a) name of the authors; b) title; c) year of publication; d) keywords; e) type of study (e.g. national or government census, questionnaire-based survey); f) type of methodological framework (e.g. quantitative methods); g) type of techniques used in the data analysis phase (e.g. statistical analyses, type of interviews, focus groups); h) quality of reporting, i.e. how thoroughly sampling methods and measurements tools are described; i) aims and type of results provided by the authors; j) type of country to refer to. Finally, all these articles will be labelled as "high relevance, medium relevance, or sufficiently relevant studies" (with respect to our purposes); 2) data coding: after being illustrated with a text string, each of the options of the aforementioned items will be summarized in a numeric code, or rather, paired off with a numerical label representative of a certain concept to refer to, e.g. " 1 " if the study is a questionnaire-based survey, " 2 " if the study is a secondary data-based survey, and so on (item 5). Item 6, which refers only to the methodological framework of the selected studies, will be represented like this: " 1 " if only quantitative methods are available, " 2 " if only qualitative methods are available, " 3 " if only mixed methods are available. This coding process transforms each item from a mere property to a variable. Such a process, which is usually referred to as "data coding", is the most 
systematic way of synthesizing and re-presenting information and empirical objects. Once all the extracted information is each assigned a code, it will be possible to carry out a statistical-descriptive analysis. Microsoft Excel 2007 will be used to enter all coded data into a searchable database.

\subsubsection{Data analysis}

We agreed to conduct a predominantly qualitative analysis, with the intention of identifying and tracing the boundaries of overlapping or unexplored conceptual areas. We will use the latest version of Maxqda to analyze all qualitative data. We will also perform basic statistical analyses, such as univariate or bivariate analyses, in order to get the frequency distribution of some of the aforementioned variables and to obtain cross tabs, e.g. referring to the comparison between HIC care systems and LIC care systems. We will also try to conduct a meta-analysis, if possible, in order to combine all the main results concerning LICs (e.g. the tendency for health inequalities to rise) with the results of other countries, especially with regard to the problem of economic impoverishment as a result of the state of health.

\subsubsection{Data synthesis and conclusions}

To summarize the main findings, all data will be represented in table form and, when appropriate, in graphic form, in a manner that aligns with the purposes of this scoping review. A narrative summary will describe how this study can be fully reproduced and how the data answers the research questions. Not too many images will be inserted, in accordance with Munn et al. The main significant differences between low-, high-, and middle-income countries, in terms of out-of-pocket medical expenses, life expectancy, and the working conditions of family caregivers, will be presented as a summary to help illustrate the different political contexts in which the problem of unpaid care can emerge (see Table 2 below). 
Table 2

The third phase of the study and related outputs

\section{CONDUCTING AND REPORTING}

\begin{tabular}{|c|c|c|c|c|c|}
\hline & Sub-phases & Description & Output 1 & Output 2 & Output 3 \\
\hline $3 A$ & $\begin{array}{l}\text { INITIAL } \\
\text { SELECTION }\end{array}$ & $\begin{array}{l}\text { Examination of a } \\
\text { broad area of } \\
\text { knowledge on the } \\
\text { issue of family } \\
\text { health care spending } \\
\text { and socioeconomic } \\
\text { deprivation }\end{array}$ & $\begin{array}{l}35 \text { items } \\
\text { selected }\end{array}$ & $\begin{array}{l}\text { Identification } \\
\text { of key concepts } \\
\text { and keywords } \\
\text { (over ten new } \\
\text { useful } \\
\text { keywords) }\end{array}$ & - \\
\hline $3 B$ & $\begin{array}{l}\text { SECOND } \\
\text { SELECTION }\end{array}$ & $\begin{array}{l}\text { a) Refinement of the } \\
\text { search process } \\
\text { through the new } \\
\text { keywords identified } \\
\text { (choosing selected } \\
\text { items); } \\
\text { b) Additional items } \\
\text { to be found } \\
\text { (advanced search) }\end{array}$ & $\begin{array}{l}\text { At present: } \\
63 \text { items } \\
\text { collected }\end{array}$ & - & - \\
\hline $3 C$ & $\begin{array}{l}\text { QUALITY } \\
\text { ASSESSMENT }\end{array}$ & $\begin{array}{l}\text { Data quality } \\
\text { assessment } \\
\text { (What kind of } \\
\text { data/results?) }\end{array}$ & $\begin{array}{l}\text { List and } \\
\text { classification } \\
\text { of the main } \\
\text { features of } \\
\text { selected } \\
\text { articles (data } \\
\text { extraction); } \\
\text { JBI data } \\
\text { extraction tool } \\
\text { (text variables) }\end{array}$ & $\begin{array}{l}\text { Codification } \\
\text { of all extracted } \\
\text { information } \\
\text { (data coding); } \\
\text { Data storage: } \\
\text { Microsoft Excel }\end{array}$ & $\begin{array}{l}\text { Identification } \\
\text { of the type of } \\
\text { analysis } \\
\text { (What kind of } \\
\text { variables can } \\
\text { be } \\
\text { analyzed?) }\end{array}$ \\
\hline $3 D$ & $\begin{array}{l}\text { DATA } \\
\text { ANALYSIS }\end{array}$ & $\begin{array}{l}\text { - Bibliographic } \\
\text { Documentary } \\
\text { Analysis (combining } \\
\text { automatic and } \\
\text { manual procedures } \\
\text { within the analysis } \\
\text { process); } \\
\text { - Meta-analysis? }\end{array}$ & $\begin{array}{l}\text { Identification } \\
\text { of any } \\
\text { conceptual } \\
\text { gap and/or } \\
\text { redundancy in } \\
\text { research or } \\
\text { basic } \\
\text { knowledge }\end{array}$ & $\begin{array}{l}\text { Identification } \\
\text { of any } \\
\text { significant } \\
\text { differences due } \\
\text { to the country in } \\
\text { which the } \\
\text { selected studies } \\
\text { were carried out }\end{array}$ & $\begin{array}{l}\text { Identification } \\
\text { of data } \\
\text { relationship } \\
\text { sets and } \\
\text { numerical } \\
\text { measures (in } \\
\text { the case of } \\
\text { meta- } \\
\text { analysis) }\end{array}$ \\
\hline $3 \mathrm{E}$ & $\begin{array}{l}\text { DATA } \\
\text { SYNTHESIS } \\
\text { AND } \\
\text { CONCLUSIONS }\end{array}$ & $\begin{array}{l}\text { - All factors linked to } \\
\text { the relationship } \\
\text { between family } \\
\text { caregiving and } \\
\text { material and social } \\
\text { deprivation will be } \\
\text { listed; } \\
\text {-Representation of } \\
\text { the level of economic }\end{array}$ & $\begin{array}{l}\text { Summary } \\
\text { tables }\end{array}$ & Graphics & \\
\hline
\end{tabular}




\section{Discussion}

We wrote about a paucity of publications on the economic burden of care for older people requiring LTC and the impact of health expenditure on their families' economic situation. However, the term "paucity" does not imply that available studies on this issue are scant. Indeed, when compared to the multitude of studies on the poor state of health of deprived older people, they are not enough for a truly exhaustive investigation: there is a compact two-way relationship between health and socioeconomic deprivation, and both directions need to be studied in-depth. We would also like to draw the attention of stakeholders and policymakers to the general issue of increasingly burdensome health costs and the difficulty of quantifying the number of people whose economic impoverishment and socio-relational deprivation are due solely or primarily to health spending. We know that many people in the world, including older adults, are incorrectly estimated to be living above the so-called 'poverty line', because of the fact that they refuse or partially refuse treatment. This specific problem can be considered as both a reason to support our study, which focuses strongly on the matter, and as a limitation, since it impedes our efforts to investigate the real magnitude of this problem. We cannot confirm with any degree of certainty the number of people who turn out to be less deprived than they appear to be, nor how many people would be officially classified as 'poor' (i.e. economically impoverished) had they received or agreed to receive appropriate assistance.

We aim to draw attention to the fact that such a thorny issue is in great need of transparency, and all the sciences involved and their research projects should contribute to the systematic construction and dissemination of knowledge and debates concerning this issue. We particularly refer to economic and socioeconomic disciplines in order to provide a useful summary of what is known about health and finance-related development data and statistics around the world, comparing high-income and lowincome countries. It is precisely because of the studies conducted in LICs, whose articles are often written in languages such as Spanish, Chinese, or Japanese, that the prevalence of the English language in the scientific field represents another limitation. Nevertheless, we expect to achieve interesting results in terms of conceptual exploration and analysis of the relationship between empirical and subjective data. Finally, we also expect to gather a large amount of data that will be used to explain any significant changes in the health welfare systems of the countries considered.

\section{Abbreviations}

PRISMA-P: Preferred Reporting Items for Systematic reviews and Meta-Analyses for Protocols 2015;

PRISMA-ScR: Preferred Reporting Items for Systematic reviews and Meta-Analyses extension for Scoping Reviews; 
JBI: Joanna Briggs Institute;

OSF: Open Science Framework;

LTC: Long-Term Care;

SES: Socio-economic Status

ADL_limitations: Activities of Daily Living limitations;

ScR: Scoping Review;

FIM: Family International Monitor;

SEreDIPE: Socio Economic deprivation related to effect of presence of Dependent older people: strategies for Innovative Policies in Europe;

LICs: Low-Income Countries;

MICs: Middle-Income Countries;

PCC: Participant, Concepts, Context

ADLs: Activities of Daily Living Scales;

Fl: Frailty Index;

HICs: High-Income Countries.

\section{Declarations}

Ethics approval and consent to participate: not applicable.

Consent for publication: not applicable.

Availability of data and materials: not applicable.

Competing interests: the authors declare that they have no competing interests.

The funders had no involvement in the design of the study, the collection, analyses or interpretation of data, the writing of the manuscript, or the decision to publish the results. Funding: the study is being supported by the Family International Monitor (FIM), the International Centre for Family Studies (CISF), and the Marie Curie European Fellowship Grant. Horizon 2020 MSCA-IF-2019 Grant Agreement No. 888102. It also partially benefits from funding for current research granted by the Italian Ministry of Health to the National Institute of Health \& Science on Ageing (IRCCS - INRCA). 
Authors' contributions: G.C. and R.M. conceived and designed the study and contributed to data collection and data management. R.M. contributed to the manuscript. All authors reviewed the paper, provided significant feedback, and approved the final manuscript. All authors have read and agreed to the published version of the manuscript.

Acknowledgements: The authors are grateful to Dr. Francesco Belletti, Director of the International Centre for Family Studies (CISF), for his suggestions on how to develop the conceptual framework of the study.

\section{References}

1. United Nations Department of Economic and Social Affairs: World Population Prospects 2019 Highlights | Multimedia Library. https://www.un.org/development/desa/publications/worldpopulation-prospects-2019-highlights.html. Accessed 4 Jun 2021

2. Population structure and ageing - Statistics Explained.

https://ec.europa.eu/eurostat/statisticsexplained/index.php?title=Population_structure_and_ageing. Accessed 4 Jun 2021

3. Percentage of population aged 80 years and over on 1 January of selected years. PNG - Statistics Explained. https://ec.europa.eu/eurostat/statisticsexplained/index.php?

title=File:Percentage_of_population_aged_80_years_and_over_on_1_January_of_selected_years. PNG. Accessed 4 Jun 2021

4. Abdi S, Spann A, Borilovic J, De Witte L, Hawley M. Understanding the care and support needs of older people: A scoping review and categorisation using the WHO international classification of functioning, disability and health framework (ICF). BMC Geriatr. 2019;19(1):1-15.

5. World Population Ageing_highlights.

https://www.un.org/development/desa/pd/sites/www.un.org.development.desa.pd/files/undesa_pd2020_world_population_ageing_highlights.pdf. Accessed 4 Jun 2021

6. Spasova S, Baeten R, Vanhercke B. Challenges in Long-Term Care in Europe. Eurohealth Observer Eurohealth, Vol. 24. 2018. https://ec.europa.eu/social/main.js. Accessed 4 Jun 2021.

7. Couto AM do, Caldas CP, Castro EAB de. Family caregiver of older adults and Cultural Care in Nursing care. Rev Bras Enferm. 2018;71(3):959-66.

8. Becqué YN, Rietjens JAC, van Driel AG, van der Heide A, Witkamp E. Nursing interventions to support family caregivers in end-of-life care at home: A systematic narrative review. Int J Nurs Stud. 2019;97:28-39.

9. Casanova G, Tur-Sinai A, Lamura G. Innovating Long-Term Care Provision in Mediterranean Welfare States: A Comparison Between Italy and Israel. J Aging Soc Policy. 2020;32(1):55-82.

10. Measuring social protection for long-term care I READ online [Internet]. https://read.oecdilibrary.org/social-issues-migration-health/measuring-social-protection-for-long-term-care_a411500aen\#page1. Accessed 4 Jun 2021 
11. Mitra S, Palmer M, Kim H, Mont D, Groce N. Extra costs of living with a disability: A review and agenda for research. Disability and Health Journal, Elsevier Inc., Vol. 10. 2017; 475-84.

12. Mayston R, Lloyd-Sherlock P, Gallardo S, Wang H, Huang Y, Montes De Oca V, et al. A journey without maps - Understanding the costs of caring for dependent older people in Nigeria, China, Mexico and Peru. PLoS One. 2017;12(8):e0182360.

13. Publications catalogue - Employment, Social Affairs \& Inclusion - European Commission. https://ec.europa.eu/social/main.jsp?catld=738\&langld=en\&publd=8106\&furtherPubs=yes. Accessed 4 Jun 2021

14. Lera J, Pascual-Sáez M, Cantarero-Prieto D. Socioeconomic inequality in the use of long-term care among European older adults: An empirical approach using the share survey. Int J Environ Res Public Health 2021;18(1):1-14.

15. Loibl C. Living in poverty: Understanding the financial behaviour of vulnerable groups. In: Economic Psychology [John Wiley and Sons Inc.] 2017; doi: 10.1002/9781118926352.ch26

16. Woo J, Yu R, Cheung K, Lai ETC. How Much Money Is Enough? Poverty and Health in Older People. J Nutr Heal Aging. 2020;24(10):1111-5.

17. Hajek A, König HH. Does the beginning and the end of income poverty affect psychosocial factors among middle-aged and older adults? Findings based on nationally representative longitudinal data. Aging Ment Heal. 2021;25(5):906-12.

18. Chen L, Cao Q. Poverty increases the risk of incident cognitive impairment among older adults: a longitudinal study in China. Aging Ment Heal. 2020;24(11):1822-7.

19. Guimarães RM, Andrade FCD. Healthy life-expectancy and multimorbidity among older adults: Do inequality and poverty matter? Arch Gerontol Geriatr. 2020;90:104157.

20. Saito M, Kondo N, Oshio T, Tabuchi T, Kondo K. Relative Deprivation, Poverty, and Mortality in Japanese Older Adults: A Six-Year Follow-Up of the JAGES Cohort Survey. Int J Env Res Public Heal. 2019; doi: 10.3390/ijerph16020182.

21. Family International Monitor: Home | Family International [Internet]. https://www.familymonitor.net. Accessed 4 Jun 2021

22. Belletti F, Casanova G. Family and Relational Poverty - Family International Monitor, 2020 Report. Murcia: UCAM; 2021

23. Lockwood C, dos Santos KB, Pap R. Practical Guidance for Knowledge Synthesis: Scoping Review Methods. Asian Nursing Research, Vol. 13. Korean Society of Nursing Science; 2019; 287-94.

24. Munn Z, Peters MDJ, Stern C, Tufanaru C, McArthur A, Aromataris E. Systematic review or scoping review? Guidance for authors when choosing between a systematic or scoping review approach. BMC Med Res Methodol. 2018;18(1):1-7.

25. Page MJ, McKenzie JE, Bossuyt PM, Boutron I, Hoffmann TC, Mulrow CD, et al. The PRISMA 2020 statement: an updated guideline for reporting systematic reviews. Syst Rev. 2021;10(1):1-11. 
26. Appendix 2.3: JBI Qualitative data extraction tool - JBI Manual for Evidence Synthesis - JBI GLOBAL WIKI.

https:// wiki.jbi.global/display/MANUAL/Appendix+2.3\%3A+JBI+Qualitative+data+extraction+tool. Accessed 4 Jun 2021

27. Casanova G, Lamura G, Principi A. Valuing and Integrating Informal Care as a Core Component of Long-Term Care for Older People: A Comparison of Recent Developments in Italy and Spain. J Aging Soc Policy. 2017;29(3):201-17.

28. Merrilees J. Activities of Daily Living. In: Encyclopedia of the Neurological Sciences [Elsevier Inc.]. $2014 ; 47-8$.

29. Lestari SK, Ng N, Kowal P, Santosa A. Diversity in the Factors Associated with ADL-Related Disability among Older People in Six Middle-Income Countries: A Cross-Country Comparison. Int J Env Res Public Heal. 2019;16(8).

\section{Supplementary Files}

This is a list of supplementary files associated with this preprint. Click to download.

- Graph1.docx

- Graph2.doc

- Additionalfile1.docx

- Additionalfile2.docx 